\title{
An Aluminophosphate Molecular Sieve with 36 Crystallographically Distinct Tetrahedral Sites
}

Jun Kyu Lee, Alessandro Turrina, Liangkui Zhu, Seungwan Seo, Daliang Zhang, Paul A. Cox, Paul A. Wright, Shilun Qiu, and Suk Bong Hong*

\section{Dedicated to Professor In-Sik Nam}

This work was supported by the NCRI (grant number 2012R1A3A- 2048833) and BK 21-plus programs through the National Research Foundation of Korea, POSCO, and the National Natural Science Foundation of China (grant numbers 91022030, 21261130584, 21201076, and 11227403). A.T. acknowledges Johnson Matthey (UK), for financial support. We thank PAL, Korea, and Diamond Light Source (DLS) (UK), for synchrotron diffraction beam time, and Prof. C. C. Tang (DLS) for assistance.

\begin{abstract}
The structure of the new medium-pore aluminophosphate molecular sieve PST-6 is determined by the combined use of rotation electron diffraction tomography, synchrotron X-ray powder diffraction, and computer modeling. PST- 6 was prepared by calcination of another new aluminophosphate material with an unknown structure synthesized using diethylamine as a structure-directing agent, which is thought to contain bridging hydroxy groups. PST-6 has 36 crystallographically distinct tetrahedral sites in the asymmetric unit and is thus crystallographically the most complex zeolitic structure ever solved.
\end{abstract}

There is a constant drive to search for new zeolitic materials because of their impact on innovation in catalysis and separation, and also on the evolution of emerging technologies.[1] Consequently, the number of zeolite framework type codes (FTCs) assigned by the Structure Commission of the International Zeolite Association (SC-IZA) continues to grow steadily. It was in the early 1980s when researchers at Union Carbide (now UOP) announced the synthesis of a family of aluminophosphate molecular sieves, abbreviated as $\mathrm{AlPO}_{4}-\mathrm{n}$, where in most cases $\mathrm{n}$ refers to a distinct framework type. [2, 3] At present, the SC-IZA recognizes $218 \mathrm{FTCs}, 44$ of which are known to occur as $\mathrm{AlPO}_{4}$-based frameworks. ${ }^{4]}$ Although $\mathrm{AlPO}_{4}$ based molecular sieves dis- play considerable structural diversity, their maximum structural complexity is much lower than that of silica-based materials. This is mainly because of the crystal-chemical requirement that $\mathrm{AlO}_{4}$ and $\mathrm{PO}_{4}$ tetrahedra must alternate, so that only even-numbered rings are present in the framework, and not odd-numbered rings like the 5-rings found in many of the most complex zeolites. The most complex zeolite structure solved is the largepore zeolite ITQ-39, the polymorphs A and B of which, with 28 crystallographically-distinct tetrahedral 
sites (T-sites), are the two most complex structures known to date. [5] By contrast, with 12 distinct Tsites, DAF-1 (DFO) is the most complex among the AlPO4-based molecular sieves. ${ }^{[6]}$

Here, we report that an $\mathrm{AlPO}_{4}$ molecular sieve denoted PST-6 (POSTECH number 6) has 36 crystallographically distinct (Al, P) atoms of equal multiplicity and 72 such $\mathrm{O}$ atoms in the asymmetric unit. Its structure has been determined and refined by a combination of electron crystallography, X-ray powder diffraction, and computer simulation. ${ }^{[7,8]}$ Leaving aside SSZ-57 (*SFV), a modulated zeolite whose "idealized" unit cell possesses 99 symmetrically independent T-sites, [9] PST-6 is crystallographically more complex than any zeolitic material known. It is also the first medium-pore material which contains a one-dimensional (1D) pore system consisting of parallel 10- and 8-ring channels.

PST- 6 was obtained by the calcination at $550{ }^{\circ} \mathrm{C}$ of another new $\mathrm{AlPO}_{4}$ phase with a bulk $\mathrm{Al} / \mathrm{P}$ ratio of unity denoted PST-5. PST-5, whose structure remains unknown, was synthesized using diethylamine (DEA), a common organic structure- directing agent yielding many zeolites and $\mathrm{AlPO}_{4}$-based molecular sieves with different framework topologies. [10,11] Its synthesis was found to be highly sensitive to the concentration of DEA in the starting synthesis gel, as well as to the type of the $\mathrm{Al}$ source employed (see Table S1 in the Supporting Information).

PST- 5 crystallizes as thin plates with a very low aspect ratio $\left(\leq 0.05\right.$; Figure S1). ${ }^{27} \mathrm{Al}$ and ${ }^{31} \mathrm{P}$ MAS NMR spectroscopies indicate that its structure is not fully tetrahedrally connected (Figure S2). Since a wide variety of $2 \mathrm{D}$ layered $\mathrm{AlPO}_{4}$ networks with $\mathrm{Al} / \mathrm{P}$ ratios of $\leq 1.0$ has long been recognized, [12] we attempted to swell PST-5 according to the same procedure as that used for the preparation of ITQ-2 [13] but without success, suggesting it is not a layered material. Calcination of PST-5 gives PST- 6 crystals with the same morphology: the individual plates remain as single crystals, implying that the transformation is topotactic (Figures S1 and S3). Thermogravimetric and differential thermal analyses reveal that PST-5 gives a large endothermic weight loss (ca. $12 \mathrm{wt} \%$ ) at temperatures up to $350{ }^{\circ} \mathrm{C}$ (Figure S4), similar to that observed for $\mathrm{AlPO}_{4}-21$ (AWO), one of the $\mathrm{AlPO}_{4}$ molecular sieves prepared using DEA in this work. The DEA molecules in both PST-5 and $\mathrm{AlPO}_{4}-21$ were found to be intact and protonated (Figures S5 and S6). Like the ${ }^{1} \mathrm{H}$ MAS NMR spectrum of $\mathrm{AlPO}_{4}-21$, the spectrum of PST-5 shows a strong ${ }^{1} \mathrm{H}$ resonance at $5.0 \mathrm{ppm}$ (Figure S7) that cannot be attributed simply to the protons of occluded water molecules. Drawing an analogy with $\mathrm{AlPO}_{4}-21$ containing 3- and 5-rings in which two adjacent trigonal-bipyramidal $\mathrm{Al}$ atoms share a hydroxyl group, ${ }^{[14]}$ it is likely that PST-5 may also contain such bridging hydroxyls. Unfortunately, our attempts to index the high resolution synchrotron powder X- ray diffraction (PXRD) pattern of PST-5 have so far been unsuccessful. 
For the PST- 6 obtained by calcination of PST-5, initial attempts to index its XRD pattern were unsuccessful. Also, we were unable to get good high-resolution transmission electron microscopy images due to the poor stability of PST- 6 in the incident $200 \mathrm{kV}$ electron beam. However, we were able to collect 3D electron diffraction data using the rotation electron diffraction (RED) tomography technique that has been used to solve the structures of nanosized, highly complex zeolites such as ITQ-

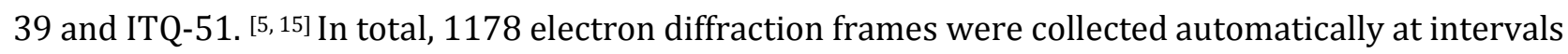
of $0.1^{\circ}$ rotation from one single PST- 6 crystal, covering $117^{\circ}\left(65 \%\right.$; -63 to $\left.54^{\circ}\right)$ of the $180^{\circ}$ required for obtaining complete 3D data.

Figure 1 shows the 3D reciprocal lattice of PST-6 reconstructed using the software RED. [16]

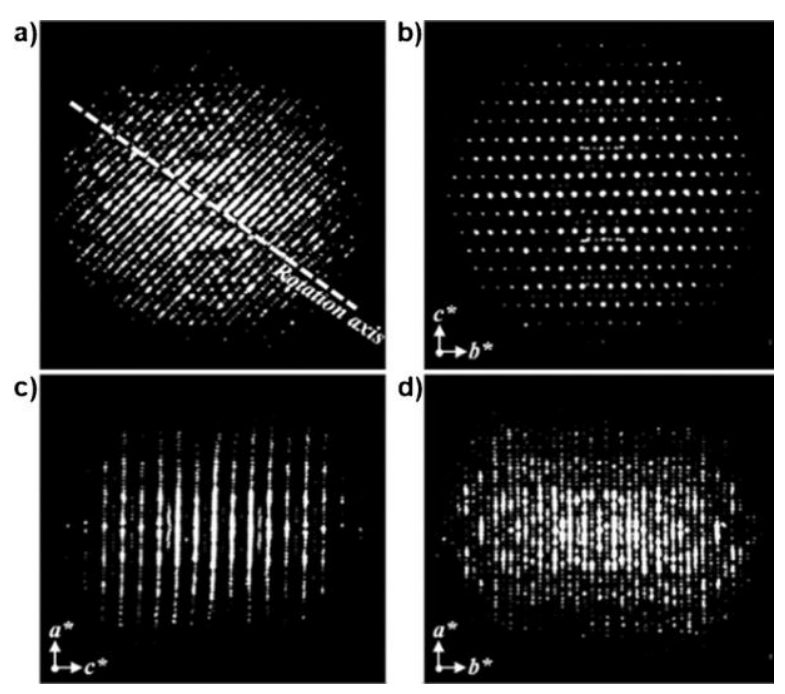

Figure 1

a) 3D reconstructed reciprocal space viewed perpendicular to the rotation axis of data acquisition. $\mathrm{b}-\mathrm{d}$ ) Projections of the 3D reconstructed reciprocal space viewed along the $a^{*}$-, $b^{*}$-, and $c^{*}$-axes, respectively.

The selected-area electron diffraction (SAED) patterns of $\mathrm{AlPO}_{4}$ phase taken along [100], [010], and [001] zone axes can be found in Figure S8. It was possible to derive approximate unit cell parameters $\left(a=39.136 \AA\right.$, $b=22.547 \AA, c=8.529 \AA$, $\alpha=90.60^{\circ}, \beta=89.45^{\circ}, \mathrm{Y}=89.88^{\circ}$; Figures S8 and S9). As shown in Figure 1, however, there are additional reflections which led us to consider a $c$-axis doubling. Because no clear evidence for these reflections is observed in the synchrotron PXRD data, on the other hand, we could not claim that their appearance is irrelevant to the dynamical effects of electron diffraction. Hence, we attempted to combine RED and PXRD data in a structure determination procedure. The unit cell parameters obtained from the electron diffraction results were used to assign indices to the peaks in the synchrotron PXRD pattern of hydrated PST-6. Although some X-ray peaks (later found to be from impurities) were not well matched, the unit cell parameters were refined to be $a=39.6500 \AA, b=22.2547 \AA, c=8.3450 \AA$ in orthorhombic (Pba2) symmetry, and a model for the $\mathrm{AlPO}_{4}$ structure was achieved using the direct methods program EXP02009.[17] After being minimized in energy using the program GULP, ${ }^{[18]}$ adopting the interatomic potentials derived by Gale 
and Henson,[19] this model was used as a starting point for the Rietveld refinement of the structures of hydrated and dehydrated PST-6.

For hydrated PST-6, the positions of adsorbed water were determined from the difference Fourier analysis and subsequently refined. Final $R_{\mathrm{wp}}$ and $R_{\mathrm{p}}$ values of 10.3 and $7.5 \%$ were achieved, respectively. The final Rietveld plot in Figure S10 provides a reasonable match between the observed and simulated PXRD patterns. The final atomic positions for hydrated PST- 6 with a refined unit cell composition I (H2O)16.0 I [ $\left.\mathrm{Al}_{72} \mathrm{P}_{72} \mathrm{O}_{288}\right]$ are listed in Table S2, and its final refined structure with $a=$ 39.5874(10) $\AA, b=22.2714(7) \AA, c=8.32807(15) \AA$ ( $P b a 2)$, is shown in Figure S11. The average Al-O and P-O bond lengths (1.72(2) and 1.53(2) $\AA$, respectively) and average O-Al-O and O-P-O angles $\left(109(3)^{\circ}\right)$ were restrained to be those expected for zeotypic $\mathrm{AlPO}_{4}$ materials (Table S3). Attempts to locate the water molecules coordinated to $\mathrm{Al}$ atoms, as suggested by the ${ }^{27} \mathrm{Al} \mathrm{MAS}$ NMR results that show the existence of a small amount of penta- and hexa-coordinated Al (Figure S2), were unsuccessful. It is likely that the water molecules are distributed in a disordered way over the framework of PST-6.

The structure of dehydrated PST-6, the ${ }^{27} \mathrm{Al}$ and ${ }^{31} \mathrm{P}$ MAS NMR spectra of which indicate a fully tetrahedrally coordinated framework, was refined against synchrotron PXRD data on a sample heated at $300^{\circ} \mathrm{C}$ under vacuum (Figure 2). A close fit to the data was achieved and details of the structure refinement can be found in Tables S4-S6. The unit cell changed to $a=38.2793(3) \AA, b=22.4638(2) \AA$, and $c=8.36197(6) \AA$ (Pba2) upon loss of water, showing a significant decrease in the $a$-axis and a smaller increase in the $b$ - and $c$ - axes (Figure S12). This can be attributed to the framework relaxation as the water molecules coordinated to some framework $\mathrm{Al}$ atoms were removed. To investigate the observation of an apparent $c$-axis doubling during the RED experiments, energy minimization of a model with a doubled cell in the $c$-axis was attempted, but no evidence for a super-structure was found.

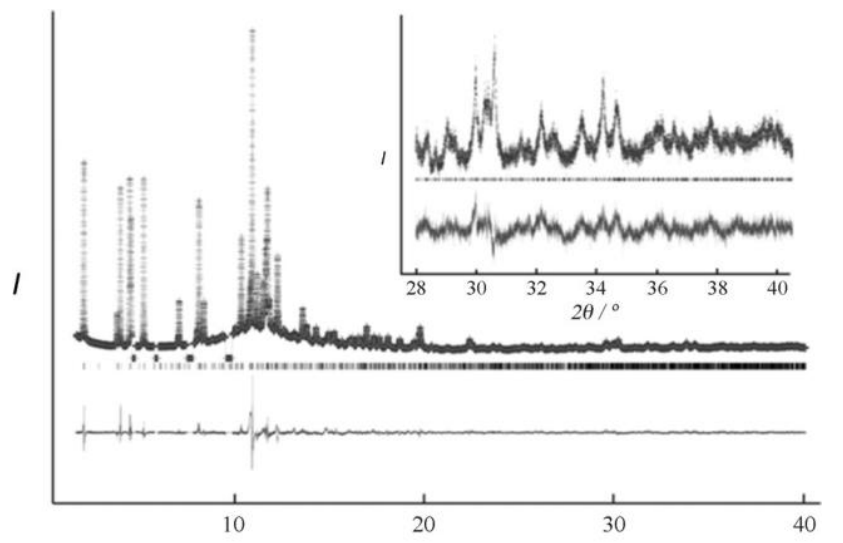

Figure 2

$2 \theta / \circ$

Rietveld plot for dehydrated PST-6: observed data (crosses), calculated fit (solid line), difference plot (lower trace). Tick marks show the positions of allowed reflections; minor impurity peaks are excluded (synchrotron, $\lambda=0.825621 \AA$ ). 
PST-6 contains a 1D pore system consisting of two parallel, elliptical 10-ring (4.2 x $6.6 \AA ̊$ ) and 8-ring $(2.3 \times 6.0 \AA ̊)$ channels running along the $c$-axis which are separated by framework walls perpendicular to the $a$ - and $b$-axes (Figure 3 and Figure S11). This material is characterized by a framework density (defined as the number of T-atoms per $1000 \AA^{3}$ ) of 19.6 which is quite high when compared with other 10-ring pore materials. ${ }^{[4]}$ We also note that the $a$-axis is perpendicular to the short dimension of the thin crystals and, structurally at least, the framework is composed of thick sheets parallel to the $b c$ plane, related by a center of symmetry across double crankshaft chains (with 4-ring projections), typical of AlPO4 molecular sieves. In fact, the Ar sorption experiments indicate that PST- 6 possesses a low micropore volume of $0.04 \mathrm{~cm}^{3} \mathrm{~g}^{-1}$ (Figure S13). Considering its unique 1D pore structure, however, PST-6 could find applications in the selective separation of small gases like $\mathrm{H}_{2}$ or $\mathrm{CO}_{2}$ based on the concept of molecular traffic control.[20]

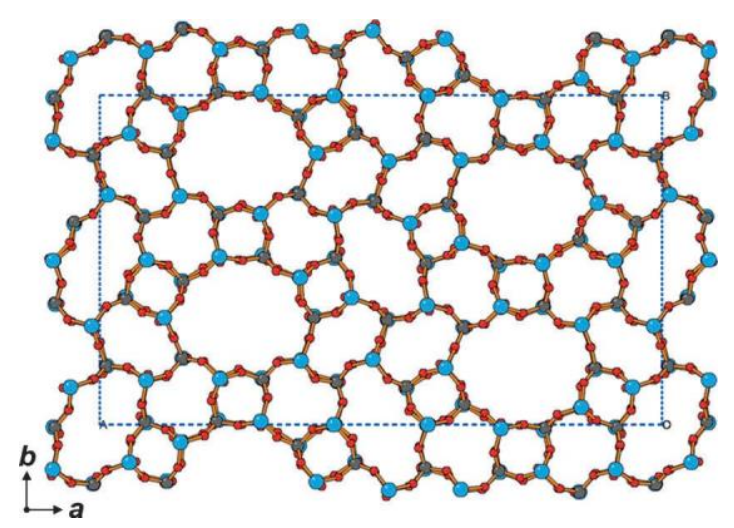

Figure 3.

Framework structure of dehydrated PST-6 with two parallel, highly elliptical 10- and 8-ring channels running along the $c$-axis. Al, light blue; P, dark gray; 0 , red.

With 36 crystallographically distinct $(\mathrm{Al}, \mathrm{P})$ atoms of equal multiplicity, the framework structure of this new $\mathrm{AlPO}_{4}$ molecular sieve is crystallographically more complex than any of the already known zeolite structures. Considering its maximum topological symmetry, that is, considering $\mathrm{Al}$ and $\mathrm{P}$ identical, it is also one of the most complex zeolitic structures known, and by far the most complex AlPO4 molecular sieve. The reason PST-6 has such a large number (18) of topologically distinct T-sites, well beyond those of other compositionally simple $\mathrm{AlPO}_{4}$ molecular sieves with even-numbered rings, is not yet clear. However, the ${ }^{27} \mathrm{Al}$ and ${ }^{31} \mathrm{P}$ MAS NMR spectra of PST-5 (Figure S2), the precursor of PST6, reveal that its framework contains penta-coordinated $\mathrm{Al}$ atoms, as well as tetrahedral $\mathrm{Al}$ atoms, and a wide range of tetrahedral $P$ environments, with a range $(-15.1--32.1 \mathrm{ppm})$ of ${ }^{31} \mathrm{P}$ chemical shifts. This range is comparable to that (-13.3--30.3 ppm) observed for $\mathrm{AlPO}_{4}-21$ which has hydroxyl groups bridging between $\mathrm{Al}$ atoms. ${ }^{[21]}$ Therefore, we think that the PST-5 framework may possess oddnumbered 3-and/or 5-rings in which Al-0-Al linkages may exist. If such is the case, it is less surprising that the precursor PST-5 has a very complex structure, features of which are inherited upon removal 
of template and bridging hydroxyls during calcination to yield a very complex zeolite structure with tetrahedral $\mathrm{Al}$ and P atoms only, that is, PST-6.

In summary, we have synthesized a new medium-pore $\mathrm{AlPO}_{4}$ molecular sieve denoted PST-6 and solved its structure by combining powder diffraction, electron crystallography, and computer modeling. PST-6 has 36 crystallographically distinct (Al, P) atoms and 72 such 0 atoms in the asymmetric unit and thus ranks as the most crystallographically complex zeolite structure known to date. It is also the first molecular sieve with a 1D system of two parallel 10- and 8-ring channels. This shows that novel, highly complex zeolite structures that contain even-numbered rings only can arise at a simple $\mathrm{AlPO}_{4}$ composition.

\section{Experimental Section}

In a typical synthesis of PST-5, $3.84 \mathrm{~g}$ of $o-\mathrm{H}_{3} \mathrm{PO}_{4}\left(85 \%\right.$, Merck) were first dissolved in $5.63 \mathrm{~g}$ of $\mathrm{H}_{2} \mathrm{O}$. This solution was added dropwise to a slurry of $6.95 \mathrm{~g}$ of aluminum isopropoxide ( $\geq 98 \%$, Aldrich) in $5.64 \mathrm{~g}$ of $\mathrm{H}_{2} \mathrm{O}$. After being stirred for $1 \mathrm{~h}, 2.45 \mathrm{~g}$ of DEA ( $99.5 \%$, Aldrich) were added. The final composition of the synthesis mixture was $2.0 \mathrm{DEA} \cdot 1.0 \mathrm{Al}_{2} \mathrm{O} 3 \cdot 1.0 \mathrm{P}_{2} \mathrm{O}_{2} \cdot 40 \mathrm{H}_{2} \mathrm{O}$. The synthesis mixture was stirred overnight at room temperature, charged into Teflon-lined $23 \mathrm{~mL}$ autoclaves, and heated at $200{ }^{\circ} \mathrm{C}$ for 5 days. PST- 6 was prepared by calcination of PST- 5 under flowing air at $550{ }^{\circ} \mathrm{C}$ for $8 \mathrm{~h}$.

Synchrotron PXRD data for PST-6 in hydrated and dehydrated forms were collected on the 9B beamline of the Pohang Acceleration Laboratory (Pohang, Korea) using monochromated X-rays $(\lambda=$ $1.54740 \AA$ ) and on the 11 beamline of the Diamond Light Source (Didcot, UK) using monochromated X-rays $(\lambda=0.825621 \AA$ A $)$, respectively. Details of the XRD measurements and Rietveld refinements can be found in Supporting Information.

\section{References}

[1] M. A. Camblor, S. B. Hong in Porous Materials (Ed.: D. W. Bruce, D. O'Hare, R. I. Walton), Wiley, Chichester, 2011, p. 265.

[2] S. T. Wilson, B. M. Lok, C. A. Messina, T. R. Cannan, E. M. Flanigen, J. Am. Chem. Soc. 1982, 104, 1146 1147.

[3] S. T. Wilson, B. M. Lok, E. M. Flanigen, US Pat 4310440, 1982. [4] C. Baerlocher, L. B. McCusker, Database of Zeolite Structures: http://www.iza-structure.org/databases/.

[5] T. Willhammar, J. Sun, W. Wan, P. Oleynikov, D. Zhang, X. D. Zou, M. Moliner, J. Gonzalez, C. Martinez, F. Rey, A. Corma, Nat. Chem. 2012, 4, 188 - 194.

[6] G. Muncaster, G. Sankar, C. R. A. Catlow, J. M. Thomas, R. G. Bell, P. A. Wright, S. Coles, S. J. Teat, W. Clegg, W. Reeve, Chem. Mater. 1999, 11, 158 - 163. 
[7] U. Kolb, T. Gorelik, C. Ktibel, M. T. Otten, D. Hubert, Ultra- microscopy 2007, 107, 507 - 513.

[8] D. Zhang, P. Oleynikov, S. Hovmçller, X. D. Zou, Z. Kristallogr.

2010, 225, $94-102$.

[9] C. Baerlocher, T. Weber, L. B. McCusker, L. Palatinus, S. I. Zones, Science 2011, 333, 1134 - 1137.

[10] R. Szostak, Handbook of Molecular Sieves, Van Nostrand Reinhold, New York, 1992.

[11] Z. Liu, X. Song, J. Li, Y. Li, J. Yu, R. Xu, Inorg. Chem. 2012, 51, 1969 - 1974.

[12] S. Oliver, A. Kuperman, G. A. Ozin, Angew. Chem. 1998, 110, 48 - 64; Angew. Chem. Int. Ed. 1998, 37, $46-62$.

[13] A. Corma, V. Fornes, S. B. Pergher, Th. L. M. Maesen, J. G. Buglass, Nature 1998, 396, 353 - 356.

[14] J. M. Bennett, J. M. Cohen, G. Artioli, J. J. Pluth, J. V. Smith, Inorg. Chem. 1985, 24, 188 - 193.

[15] R. Martfnez-Franco, M. Moliner, Y. Yun, J. Sun, W. Wan, X. D. Zou, A. Corma, Proc. Natl. Acad. Sci. USA 2013, 110, 3749 - 3754.

[16] W. Wan, J. Sun, J. Su, S. Hovmçller, X. D. Zou, J. Appl. Crystallogr. 2013, 46, 1863 - 1873.

[17] A. Altomare, M. Camalli, C. Cuocci, C. Giacovovazzo, A. Moliterni, R. Rizzi, J. Appl. Crystallogr. 2009, 42, $1197-1202$.

[18] J. D. Gale, A. L. Rohl, The General Utility Lattice Program, Mol. Simul. 2003, 29, 291 - 341.

[19] J. D. Gale, N. J. Henson, J. Chem. Soc. Faraday Trans. 1994, 90, 3175 - 3179.

[20] R. Harish, D. Karevski, G. M. Schtitz, J. Catal. 2008, 253, 191 - 199.

[21] R. Jelinek, B. F. Chmelka, Y. Wu, P. J. Grandinetti, A. Pines, P. J. Barrie, J. Klinowski, J. Am. Chem. Soc. 1991, 113, 4097 - 4101. 